\title{
Molecular-dynamics simulations of the dynamical excitations in commensurate submonolayer films of nitrogen molecules on graphite
}

\author{
F. Y. Hansen \\ Department of Physical Chemistry, Technical University of Denmark, FKI-206-DTU, DK-2800, Lyngby, Denmark \\ L. W. Bruch \\ Department of Physics, University of Wisconsin-Madison, Madison, Wisconsin 53706 \\ H. Taub \\ Department of Physics and Astronomy, University of Missouri-Columbia, Columbia, Missouri 65211
}

(Received 2 October 1995; revised manuscript received 17 June 1996)

\begin{abstract}
The dynamics of commensurate submonolayer solids of $\mathrm{N}_{2}$ molecules adsorbed on the basal planes of graphite have been studied using molecular-dynamics simulations. The calculations yielded the temperature dependence of the Brillouin-zone-center gap in the acoustic-phonon branches, for comparison with inelastic neutron-scattering experiments on the submonolayer solid. The calculated frequency gap was the same in submonolayer and monolayer films at low temperatures. At intermediate temperatures, the diffusive molecular motion associated with the presence of vacancies caused the gap mode to be less clearly defined in the coherent scattering function. Diffusion constants are calculated at submonolayer coverages, and temperatures up to $40 \mathrm{~K}$ for a population of molecules identified as mobile. [S0163-1829(96)07243-8]
\end{abstract}

Monolayer and submonolayer films of molecular nitrogen adsorbed on the basal planes of graphite have drawn intense experimental $^{1-7}$ and theoretical ${ }^{8-15}$ interest because of the wide range of surface phenomena that occur there. One emphasis has been on the evolution of structural and dynamical properties with temperature and their correlation with disordering transitions. In the submonolayer regime, the melting temperature increases from $\sim 48 \mathrm{~K}$ at 0.5 layers to $\sim 75 \mathrm{~K}$ at 1.0 layers ${ }^{16}$ in the simulations, this has been correlated with differing mechanisms of vacancy production in the solid film. We previously reported molecular-dynamics simulations of excitations in the 1.0-layer film and of the melting of submonolayer films. ${ }^{14,15}$ Here we extend the methodology to an analysis of the dynamics of the submonolayer solid film with thermally activated vacancies. $8,12,13$

The acoustic branches of a commensurate monolayer solid have a frequency gap at the center of its Brillouin zone that is primarily determined by the "corrugation" or lateral variation in the molecule-substrate potential. The inelastic neutron-scattering experiments on the nitrogen film to measure the zone-center gap ${ }^{6,7}$ had an uncertainty in the calibration of coverage. Measurements were said to be conducted on a complete monolayer film, but the reported melting point of $\sim 50 \mathrm{~K}$ corresponds to submonolayer coverage. Previous calculations of the gap frequency of commensurate $\mathrm{N}_{2} /$ graphite evaluated the coherent scattering function only for a complete monolayer. ${ }^{10,14}$ In the case of $\mathrm{Kr} /$ graphite, it was calculated for a submonolayer patch without defects. ${ }^{17} \mathrm{We}$ now calculate the Brillouin-zone-center frequency gap for solid submonolayer $\mathrm{N}_{2}$ films at temperatures where there are significant defect populations. The resulting similarity in the temperature dependence of the zone-center gap in the submonolayer and monolayer reduces the uncertainty in the interpretation of the neutron-scattering experiments. 6,7
The series of molecular-dynamics (MD) (Refs. 8 and 15) and Monte Carlo ${ }^{13}$ simulations on the commensurate monolayer and submonolayer $\mathrm{N}_{2}$ films showed that thermally excited vacancies in the films are an important source of the free volume needed for the melting transition. These thermally excited vacancies in the submonolayer solid film also complicate the determination of the frequency spectra by enhancing the diffusive intraplanar motion of molecules. We extend previous characterizations of the defect motions by analyzing mean-square displacements and molecular trajectories to supplement available information on mobile populations and diffusion constants.

The interaction model and the isothermal constrained molecular-dynamics algorithm are the same as in our previous papers. ${ }^{14,15}$ The submonolayer patches again are in the form of strips, Refs. 11 and 15. The systems are typically propagated for 500 ps and for up to 800-1100 ps near a phase transition. Time-correlation functions for both single molecule and collective motions are calculated using a 200-ps time series of data.

The collective motions of the monolayer solid are analyzed using the intermediate coherent scattering function and its Fourier transform, the coherent scattering function. We show results for the center-of-mass one-phonon forms; parallel calculations with the atomic coordinates give similar, but noisier, spectra. The incoherent scattering function is also examined in a frequency range which would correspond to quasielastic scattering.

In the case of the zone-center gap, even with a 200-ps time series of molecular-dynamics data, a "baseline", subtraction $^{14}$ in the intermediate scattering function is needed in order to eliminate an elastic-scattering component that produces large truncation satellites in the Fourier spectrum, and to enhance the signature of the inelastic peak. For a solid in which all the molecules are localized, the subtrac- 
tion is unambiguous; but, when there is significant solid-state diffusion, the procedure becomes problematic. We find it useful to divide the adsorbed molecules into two classes, termed mobile and localized, which make distinct contributions to both the scattering functions and the mean-square displacements.

Voronoi constructions ${ }^{15}$ and trajectory plots (not shown here) for the submonolayer commensurate solid showed that, as the temperature increases, defect structures develop first in the edge region of a patch and then in the patch interior. In terms of the molecules, some leave the carbon hexagon which they initially occupy on the graphite surface, while others, especially in the patch interior, remain in the original carbon hexagon for long periods and have a mean-square displacement similar to that found in the monolayer film at the same temperature. We use such differences as the basis of a "dynamical" definition: in a given 200-ps time sequence, a localized molecule is one which does not leave its initial carbon hexagon, and a mobile molecule is one which makes at least one transition between hexagons. ${ }^{18}$ The baseline subtraction for the intermediate coherent scattering function of the localized molecules is straightforward. Molecular trajectory plots show that this definition of the mobile population includes molecules in the interior of a high-coverage submonolayer solid which have a mobility as a result of vacancy motions.

The free edge regions of the submonolayer patches cause only a very modest downshift in the frequency spectra of the translational and rotational velocity autocorrelation functions as compared to those for the monolayer film at the same temperature. Just below the submonolayer melting temperature, there is a diffusive component in the spectra which arises from the mobility of some of the molecules.

Diffusion constants $D$ are derived from the mean-square molecular center-of-mass displacement using

$$
D_{2}(\tau) \equiv \sum_{j=1}^{N^{\prime}}\left\langle\left[\mathbf{R}_{j}\left(t_{0}+\tau\right)-\mathbf{R}_{j}\left(t_{0}\right)\right]^{2}\right\rangle / N^{\prime} \approx 4 D \tau .
$$

The brackets denote an average over initial times $t_{0}$, the sum runs over the $N^{\prime}$ mobile molecules, and $D$ is fit to the variation after an initial transient. Results for the mean-square displacement of the $\rho=0.5$ film at $30 \mathrm{~K}$, just below the melting transition at $\sim 34 \mathrm{~K}$, are shown in Fig. 1. The mobile populations and the associated diffusion constants are listed in Table I for two coverages and several temperatures. As the temperature increases, the motions become more nearly a uniform diffusion, but the diffusion constants remain distinctly smaller than those for fluids ${ }^{12}$ until the layer melts. The fraction of mobile molecules is larger than might have been expected from Voronoi constructions on instantaneous configurations, ${ }^{15}$ and reflects time variations associated with edge roughness.

We use calculations of the Fourier transform of the intermediate coherent scattering function evaluated at wave vectors corresponding to reciprocal-lattice vectors of the monolayer solid to determine the dependence of the Brillouinzone-center frequency gap on coverage and temperature. Spectra from calculations which include all the molecules show inelastic scattering peaks close to those for the localized population, but with quite a large "background." The

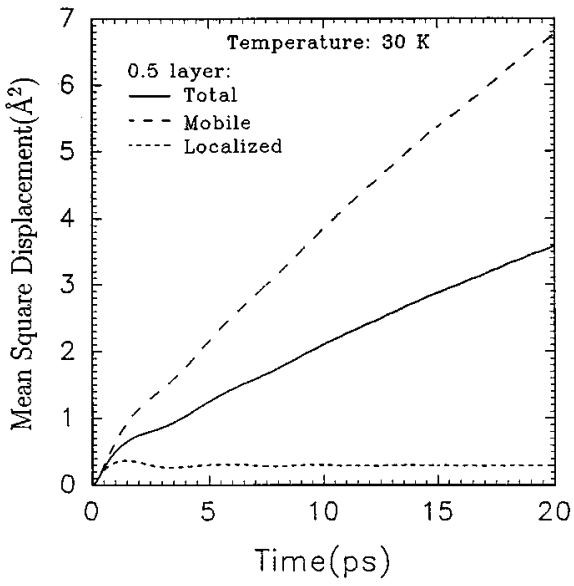

FIG. 1. The mean-square displacement of the nitrogen molecules in a 0.5-layer film as a function of time at $30 \mathrm{~K}$. The separated mean-square displacements of localized and mobile molecules are also shown. The population of mobile molecules, as defined in the text, is given in Table I. The mean-square displacement of a localized molecule is the same as that for $\rho=1.0$. The total displacement curve is a sum of these contributions weighted with the respective mole fractions.

background problem is present in calculations with the full intermediate scattering function and in the one-phonon approximation to that function. The results presented here are based on the center-of-mass one-phonon functions for the localized-molecule population. Then the inelastic peak is clearly visible.

TABLE I. Diffusion constants for mobile molecules. Numbers of mobile molecules and the corresponding two-dimensional diffusion constants $D$, in units of $10^{-5} \mathrm{~cm}^{2} / \mathrm{s}$, are listed as a function of temperature $T$ in $\mathrm{K}$ and fractional coverage $\rho$. Values of $D$ are derived using Eq. (1) for the mean-square displacements of mobile molecules with $\tau$ in the range $1-20 \mathrm{ps}$ and $t_{o}$ averaged over a 200-ps time block.

\begin{tabular}{lcccc}
\hline \hline$T$ & \multicolumn{2}{c}{$\rho=0.5^{\mathrm{a}}$} & \multicolumn{2}{c}{$\rho=0.929^{\mathrm{b}}$} \\
& $N_{\text {mobile }}$ & $\mathrm{D}$ & $N_{\text {mobile }}$ & $\mathrm{D}$ \\
\hline & & & & \\
20 & 15 & 0.14 & 16 & 0.13 \\
25 & 30 & 0.16 & & \\
30 & 57 & 0.81 & 42 & 0.27 \\
35 & 112 & $4.2^{\mathrm{c}}$ & & \\
40 & & & 135 & 0.55 \\
\hline \hline
\end{tabular}

${ }^{a}$ Calculations for strip geometry 7/14 of Ref. 15, i.e., 112 molecules in the periodic cell. The number of localized molecules equals 112 $-N_{\text {mobile }}$. For $T \leqslant 30 \mathrm{~K}$, trajectory plots show that the mobile molecules are concentrated near the geometric edge of the strip.

${ }^{b}$ Calculations for strip geometry 13/14 of Ref. 15, i.e., 208 molecules in the periodic cell. The number of localized molecules equals $208-N_{\text {mobile }}$. Trajectory plots show that at $30 \mathrm{~K}$ the mobile molecules are concentrated near the geometric edges of the strip and are not present in its interior, but at $40 \mathrm{~K}$ they have penetrated the interior.

${ }^{\mathrm{c}}$ The width of the Lorentzian component in Fig. 4 gives a value 2-4 in these units. 


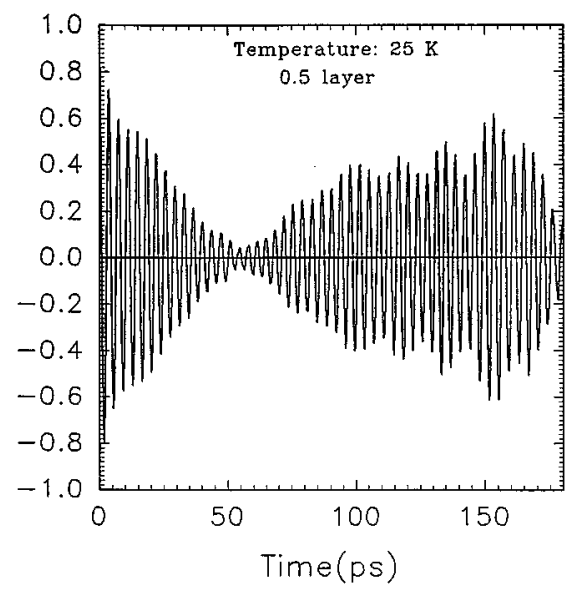

(a)

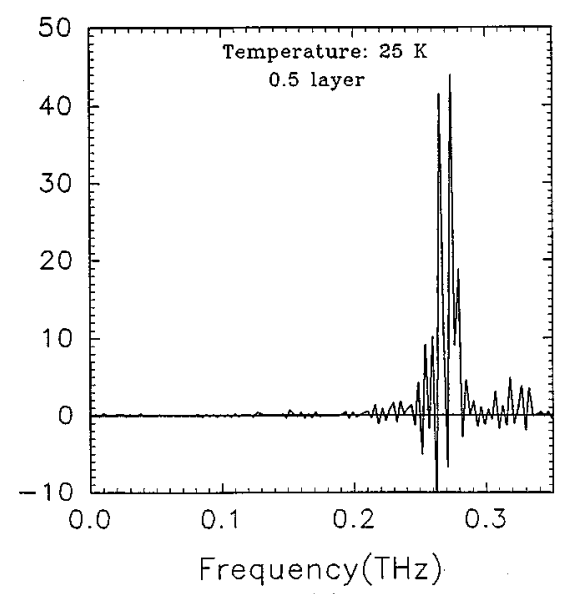

(b)

FIG. 2. The modified center-of-mass one-phonon intermediate coherent scattering function for localized molecules in the $\rho=0.5$ film at $25 \mathrm{~K}$ for the reciprocal-lattice vector $1.703 \hat{\mathbf{x}} \AA^{-1}$. (a) Timecorrelation function. (b) Fourier transform.

We looked in the coherent scattering function for a signature of quasielastic scattering from the diffusive motion of mobile molecules in the solid, but could find no clear evidence of it. Apparently, the diffusion constants, as listed in

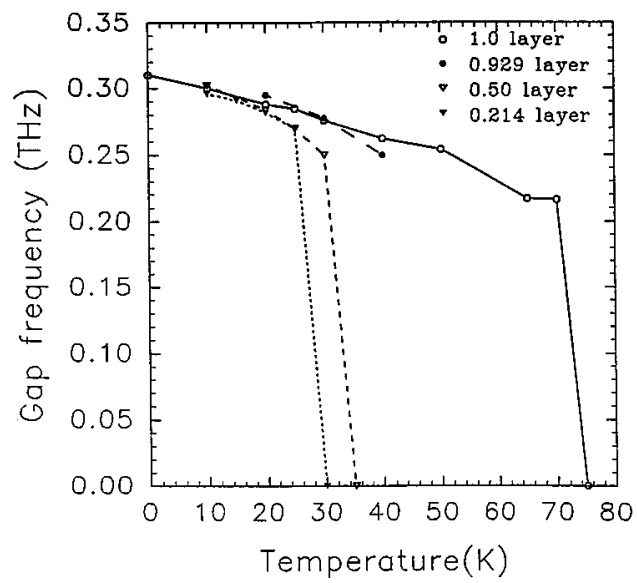

FIG. 3. The Brillouin-zone-center gap frequencies at four coverages as a function of temperature for the reciprocal-lattice vector $1.703 \hat{\mathbf{x}} \AA^{-1}$. Values for the reciprocal lattice vector $2.905 \hat{\mathbf{y}} \AA^{-1}$ are quite similar.

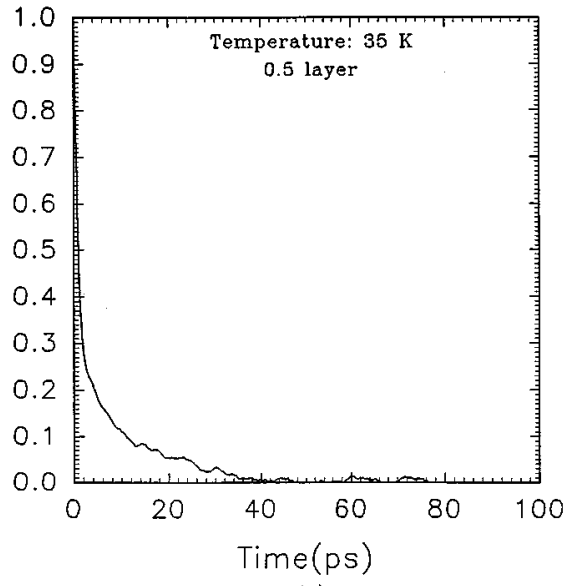

(a)

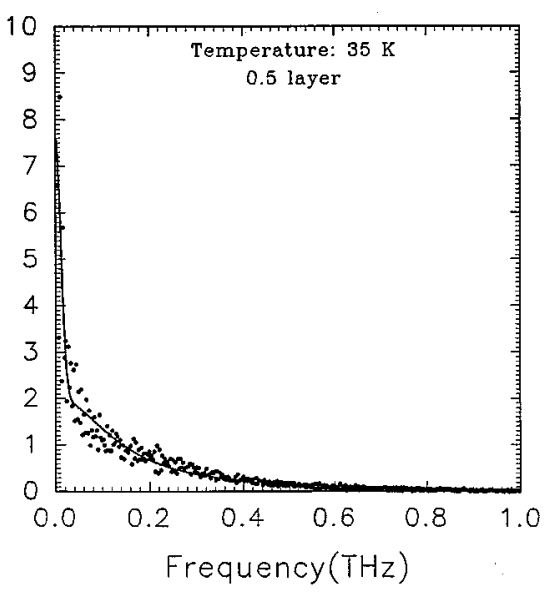

(b)

FIG. 4. Incoherent scattering function for the 0.5-layer fluid film at $35 \mathrm{~K}$, calculated for $\kappa=1.703 \hat{\mathbf{x}} \AA^{-1}$. (a) Time-correlation function. (b) Fourier transform, showing a large quasielastic component. The solid line is a fit to a superimposition of Gaussian and Lorentzian functions both centered at zero frequency. The dots are the MD results. A diffusion constant in the range $2-4 \times 10^{-5} \mathrm{~cm}^{2} / \mathrm{s}$ is inferred from the width of the Lorentzian.

Table I, are too small for there to be a signal that rises above the computational background.

Figure 2 shows the one-phonon approximation, as defined in Eq. (15) of Ref. 14, to the modified center-of-mass intermediate coherent scattering function and its Fourier transform for the localized molecules in the $\rho=0.5$ film at $25 \mathrm{~K}$; the wave vector is $\kappa=1.703 \hat{\mathbf{x}} \AA^{-1}$, a reciprocal-lattice vector of the commensurate lattice. The Fourier transform in Fig. 2(b) shows a peak at about $0.28 \mathrm{THz}$, which is the Brillouin-zone-center frequency gap for translational motion along $\hat{\mathbf{x}}$. The time dependence has a beating phenomenon superimposed on a nearly harmonic oscillation; we have seen such beating under a great variety of conditions for both the submonolayer and monolayer solids.

The calculations at 10 and $20 \mathrm{~K}$ also give time-correlation functions with a beating pattern superimposed on a nearly sinusoidal function, and spectral peaks in the $0.28-0.29-\mathrm{THz}$ range, close to the results for $\rho=1.0$ at the same temperatures. ${ }^{14}$ As noted by Shrimpton and Steele, ${ }^{17}$ it is difficult to achieve thermal equilibrium populations of the long-lived zone-center phonons on the time scale of a 
molecular-dynamics simulation. We found variations in the amplitude of the nearly harmonic oscillation over a $500 \mathrm{ps}$ time series without reaching the equipartition averages for the low-temperature solid anticipated from the sum-rule of Hansen and Klein. ${ }^{19}$ A model with a dynamic substrate probably would resolve this problem through the radiative damping mechanism of Hall, Mills, and Black. ${ }^{20}$

The gap frequency for the zone-center mode, derived from data such as those shown in Fig. 2, is plotted in Fig. 3 as a function of temperature. Up to $25 \mathrm{~K}$, the gap for $\rho=0.5$ is equal to that for $\rho=1.0$ at the same temperature. Differences from the $\rho=1.0$ results begin to develop as the melting temperature is approached. The calculations at $30 \mathrm{~K}$, just $4 \mathrm{~K}$ below the melting temperature of the $\rho=0.5$ film, show a broadening of the spectral peak, a decrease in the peak frequency, and a lowering of its intensity relative to the rest of the spectrum.

The melting temperature of the $\rho=0.929$ film is near 60 $\mathrm{K}$ for our model. At $40 \mathrm{~K}$, vacancies permeate the solid as a result of molecular diffusive motion, as evidenced by the data in Table I. However, the modified one-phonon scattering function for $\kappa=1.703 \hat{\mathbf{x}} \AA^{-1}$ at $40 \mathrm{~K}$ remains similar to that for $\rho=1.0$, and has a spectral peak centered at about $0.24 \mathrm{THz}$. Results for the $\rho=0.929$ film are included in Fig. 3 , and show that the gap frequency of this film remains close to that for $\rho=1.0$ up to $40 \mathrm{~K}$. As for $\rho=0.5$, an accurate determination of the gap frequency becomes increasingly difficult at higher temperatures.

Finally, as a rough check on the consistency of the interpretation of the diffusive motion in the $\rho=0.5$ fluid at $35 \mathrm{~K}$, just $1 \mathrm{~K}$ above the melting point, the incoherent (self-) scattering function and its Fourier transform were evaluated for several wave vectors. The results for $\kappa=1.703 \hat{\mathbf{x}} \AA^{-1}$ are shown in Fig. 4. The frequency spectrum has been fit to superpositions of a Gaussian, representing an elastic component, and a Lorentzian, representing a diffusive component, both centered at zero frequency. From the width of the Lorentzian we calculate a diffusion constant in the range of
$2-4 \times 10^{-5} \mathrm{~cm}^{2} / \mathrm{s}$. This is a quite satisfactory agreement with the diffusion constant in Table I derived from the meansquare displacement, considering the untested assumptions involved in the choice of the fitting functions.

In summary, the analysis of the molecular motions presented here supports an identification of two successive temperature regimes for vacancy effects in the submonolayer solid film. At low temperatures the defects are concentrated at patch edges, and, for high submonolayer coverages, there is an intermediate-temperature range where defects permeate the patch interior. The relatively small diffusion constants of the $\rho=0.929$ film are consistent with the formation of a defect solid ${ }^{21}$ at the melting temperature of the $\rho=0.5$ film. The diffusion constants for the mobile molecules in this solid are large enough to give recognizable effects in the meansquare displacements averaged over the film, but are small enough that we have not been able to demonstrate a signature in the correlation functions governing quasielastic scattering.

The frequency gap is insensitive to coverage in the range $\rho=0.5-1.0$ until close to the melting temperature. Indeed, one expects the gap to be determined primarily by the lateral restoring force exerted on the solid adlayer by the corrugation of the molecule-substrate potential. Within $10 \mathrm{~K}$ of the melting temperature, there is an intensity loss in the gapfrequency peak of the scattering functions, and the gap mode is less clearly defined. Knowledge of the exact coverage at which the neutron-scattering experiments ${ }^{6,7}$ were performed is therefore not crucial for analyses which use the gap frequency measured at low temperatures to determine the corrugation.

This work was partially supported by the National Science Foundation under Grant No. DMR-9314235 (H.T.) and Nos. DMR-9120199 and DMR-9423307 (L.W.B.) and by The Danish Natural Science Foundation (F.Y.H.). L.W.B. thanks the Fysisk-Kemisk Institut and the Technical University of Denmark for hospitality during the period this work was completed.
${ }^{1}$ T. T. Chung and J. G. Dash, Surf. Sci. 66, 559 (1977).

${ }^{2}$ R. D. Diehl and S. C. Fain, Jr., Surf. Sci. 125, 116 (1983).

${ }^{3}$ A. D. Migone, H. K. Kim, M. H. W. Chan, J. Talbot, D. J. Tildesley, and W. A. Steele, Phys. Rev. Lett. 51, 192 (1983).

${ }^{4}$ M. H. W. Chan, A. D. Migone, K. D. Miner, and Z. R. Li, Phys. Rev. B 30, 2681 (1984).

${ }^{5}$ M. H. W. Chan, in Phase Transitions in Surface Films 2, edited by H. Taub, G. Torzo, H. J. Lauter, and S. C. Fain, Jr. (Plenum, New York, 1991), pp. 1-10.

${ }^{6}$ H. J. Lauter, V. L. P. Frank, H. Taub, and P. Leiderer, in Proceedings of the 19th International Conference on Low Temperature Physics, edited by D. S. Betts [Physica B 165 \& 166, 611 (1990)].

${ }^{7}$ F. Y. Hansen, V. L. P. Frank, H. Taub, L. W. Bruch, H. J. Lauter, and J. R. Dennison, Phys. Rev. Lett. 64, 764 (1990).

${ }^{8}$ J. Talbot, D. J. Tildesley, and W. A. Steele, Mol. Phys. 51, 1331 (1984)

${ }^{9}$ R. M. Lynden-Bell, J. Talbot, D. J. Tildesley, and W. A. Steele, Mol. Phys. 54, 183 (1985); D. J. Tildesley and R. M. Lynden-
Bell, J. Chem. Soc. Faraday Trans. II 82, 1605 (1986).

${ }^{10}$ G. Cardini and S. F. O’Shea, Surf. Sci. 154, 231 (1985).

${ }^{11}$ Y. P. Joshi and D. J. Tildesley, Mol. Phys. 55, 999 (1985).

${ }^{12}$ A. V. Vernov and W. A. Steele, Langmuir 2, 606 (1986).

${ }^{13}$ R. D. Etters, B. Kuchta and J. Belak, Phys. Rev. Lett. 70, 826 (1993); M. Roth and R. D. Etters, Phys. Rev. B 44, 6581 (1991); R. D. Etters, M. W. Roth, and B. Kuchta, Phys. Rev. Lett. 65, 3140 (1990); B. Kuchta and R. D. Etters (unpublished).

${ }^{14}$ F. Y. Hansen and L. W. Bruch, Phys. Rev. B 51, 2515 (1995).

${ }^{15}$ F. Y. Hansen, L. W. Bruch, and H. Taub, Phys. Rev. B 52, 8515 (1995).

${ }^{16}$ All coverages in this paper are given in terms of fractions $\rho$ of a vacancy-free $\sqrt{3} \times \sqrt{3}$ commensurate monolayer.

${ }^{17}$ N. D. Shrimpton and W. A. Steele, Phys. Rev. B 44, 3297 (1991).

${ }^{18}$ There is arbitrariness in the choice of time scale and in the amount of motion required to qualify a molecule to be mobile. Indeed, a large variation in populations was observed when the time span was changed from 20 to $100 \mathrm{ps}$, but then the populations became almost stationary and had only a weak variation as 
the time span was increased to $200 \mathrm{ps}$. Increasing the amount of required motion reduced the mobile population, as expected, but the population identified on the basis that there is one transition between hexagons already has a diffusive motion (i.e., a linear time variation for the center-of-mass mean-square displacement).

${ }^{19}$ J. P. Hansen and M. L. Klein, Phys. Rev. B 13, 878 (1976).

${ }^{20}$ B. Hall, D. L. Mills, and J. E. Black, Phys. Rev. B 32, 4932 (1985).
${ }^{21}$ The term defect solid has been used to refer to a solid that has a large enough concentration of thermally excited vacancies that there is a significant solid-state diffusion constant. However, the diffusion constant remains smaller than that for a fluid, and the diffraction peaks remain large and narrow. See Talbot, Tildesley, and Steele (Ref. 8) for an early comment on formation of a defect solid, and Vernov and Steele (Ref. 12) for an attempt to distinguish the defect solid from a fluid phase at the onset of multilayer condensation in $\mathrm{N}_{2}$ /graphite near $75 \mathrm{~K}$. 